\title{
EDITORIAL
}

\section{La Santé Intégrative en orthodontie, pour améliorer la rééducation myofonctionnelle orofaciale}

\author{
Alain Toledano \\ Médecin cancérologue-radiothérapeute, \\ Directeur de chaire en Santé Intégrative \\ au conservatoire national \\ des arts et métiers \\ Président de l'Institut Rafaël
}

Quoi de plus à propos que d'aborder la démarche en santé intégrative en préface d'une revue consacrée à la rééducation myofonctionnelle orofaciale?

Les orthodontistes d'aujourd'hui sont des acteurs de santé incontournables, se préoccupant aussi bien des problématiques esthétiques que fonctionnelles. Ils prennent en charge de nombreuses fonctions majeures comme l'occlusion, la déglutition, la mastication, ou encore le sommeil... Cela nécessite d'avoir une approche globale et pas seulement technique. La collaboration avec les kinésithérapeutes, les orthophonistes, les médecins du sommeil, les chirurgiens maxillo-faciaux et autres praticiens, est déjà ancrée dans l'exercice routinier. Nous pensons que l'orthodontie peut être un des fers de lance de la santé intégrative qui permettra de réformer notre système sanitaire français.

La santé est le premier sujet de préoccupation des Français. Alors que l'État lui consacre plus de 260 milliards d'euros chaque année, soit plus de $11 \%$ de son PIB, les grandes réformes se font attendre. La moitié des 400 millions de consultations médicales réalisées en France chaque année concerne la gestion de symptômes de maladies chroniques. Nous y répondons en prescrivant des médicaments dans plus de $90 \%$ des cas. Hormis le fait que nous jetons à la poubelle une boîte sur deux, soit 7 milliards d'euros par an, cela appelle à une véritable réflexion de tous les acteurs de santé sur la manière de soigner et de prendre soin.

L'orthopédie dento-faciale est une plaque tournante du système de santé. En prenant en charge plus de 2 millions de français chaque année, sa capacité à changer les mentalités est immense. Ses populations cibles sont variées, le fait qu'il s'agisse majoritairement d'enfants et de parents permet d'envisager de véritables leviers d'éducation sanitaire, notamment en termes de prévention.

\section{Qu’est-ce que la santé intégrative?}

C'est un changement de prisme dans la considération de la santé, qui consiste à ne pas seulement la considérer comme l'absence de maladie, mais aussi au travers de ses composantes psychologique, sociale, émotionnelle, sexuelle, environnementale... 
Ne plus traiter une pathologie mais un individu de façon globale, en lui donnant du temps et de l'attention, et en intégrant l'ensemble des autres disciplines disponibles pour compléter l'offre de soins.

Pour plus d'efficience et mieux considérer humainement chaque patient, l'approche en santé intégrative doit compléter la vision scientifique acquise qui accompagne chacune de nos disciplines médicales. II s'agit d'intégrer chaque discipline pour considérer l'hétérogénéité des patients et de leurs besoins, et les évaluer. Ainsi, nous pourrions imaginer plusieurs études à mener pour améliorer les problématiques myofonctionnelles, dans des disciplines aussi variées que la nutrition, I'acupuncture, I'ostéopathie, la sophrologie, I'hypnose, etc.

La culture et le spectre vaste d'activités de l'orthodontie doivent donner l'exemple dans la personnalisation des approches thérapeutiques et l'intégration de la pluridisciplinarité dans l'exercice.

On définit guérir comme le retour à un état de bien-être physique, mental et social, gageons que l'approche multidimensionnelle du soin en orthodontie dépasse la technique, et permette de réécrire l'histoire de la guérison en valorisant l'approche en santé intégrative.

\section{NDLR :}

